\title{
HEATING OF THE AHLFORS-BEURLING OPERATOR, AND ESTIMATES OF ITS NORM
}

\author{
A. VOLBERG AND F. NAZAROV
}

\begin{abstract}
A new estimate is established for the norm of the Ahlfors-Beurling transform $T \varphi(z):=\frac{1}{\pi} \iint \frac{\varphi(\zeta) d A(\zeta)}{(\zeta-z)^{2}}$ in $L^{p}(d A)$. Namely, it is proved that $\|T\|_{L^{p} \rightarrow L^{p}} \leq$ $2(p-1)$ for all $p \geq 2$. The method of Bellman function is used; however, the exact Bellman function of the problem has not been found. Instead, a certain approximation to the Bellman function is employed, which leads to the factor 2 on the right (in place of the conjectural 1 ).
\end{abstract}

\section{$\S 0$. INTRODUCTION}

\section{Notation.}

$:=$ means "is equal by definition";

$x:=\left(x_{1}, x_{2}\right)$;

$D(x, R)$ is the disk centered at $x$ and of radius $R$;

$k(x, t):=\frac{1}{4 \pi t} e^{-\frac{\|x\|^{2}}{4 t}}$ is the heat kernel on the plane;

$\mathcal{D}$ is a collection of dyadic intervals.

Main objects and results. The main object in this paper is the Ahlfors-Beurling operator given by

$$
T \varphi(z):=\frac{1}{\pi} \iint \frac{\varphi(\zeta) d A(\zeta)}{(\zeta-z)^{2}}
$$

Here $d A$ denotes area Lebesgue measure on $\mathbb{C}$. Our goal is to present a new estimate of the norm of $T$. This estimate falls short of the proof of the well-known conjecture saying that

$$
\|T\|_{L^{p} \rightarrow L^{p}}=p-1, \quad p \geq 2 .
$$

Here we show that $\|T\|_{L^{p} \rightarrow L^{p}} \leq 2(p-1)$ for all $p \geq 2$, which is two times worse than (0.1). The estimate $\|T\|_{L^{p} \rightarrow L^{p}} \leq 4(p-1)$ was established in 3]. After the first preprint version of the present paper appeared, Rodrigo Bañuelos and Pedro MéndezHernández 9] informed us that they also managed to improve 4 to 2 by modifying the methods used in [3].

Actually, this problem has a long history, and it has been reappearing in many papers on the regularity of quasiconformal homeomorphisms and quasiregular maps. Essentially, the $L^{p}$-theory of quasiregular mappings was started with the work of B. Bojarski [5, 6]. Later, this subject came under intensive investigation. In particular, the best integrability of $K$-quasiconformal mappings and the problem (dual in a sense; see [28]) on the minimal regularity of quasiregular mappings were discussed in many papers. Here we mention [15]-[17], [18, 19], [22]-[24], [27, 28]. The best integrability result was established finally

2000 Mathematics Subject Classification. Primary 42B20, 42C15, 42A50, 47B35.

Partially supported by the NSF grant DMS 0200713. 
in [2]. The best minimal regularity result was obtained recently in [30. The method of [30] will be applied in the present paper to establish the inequality

$$
\|T\|_{L^{p} \rightarrow L^{p}} \leq 2(p-1), \quad p \geq 2 .
$$

By the same method, it is possible to prove that

$$
\left\|\left(\sum_{j, k=1}^{2}\left|\frac{\partial^{2} f}{\partial x_{j} \partial x_{k}}\right|^{2}\right)^{1 / 2}\right\|_{p} \leq \sqrt{2} p\|\Delta f\|_{p}, \quad f \in W_{2}^{p}, \quad p \geq 2,
$$

which is better than in [24].

\section{$\S 1$. Consequences of the "( $p-1)$-estimate"}

Let us formulate analytic and geometric consequences of the elimination of the factor 2 in (0.2). We are dealing with (local) solutions of the Beltrami equation

$$
f_{\bar{z}}-\mu f_{z}=0 .
$$

We ask two questions.

1) Suppose $\|\mu\|_{\infty}=k<1$. If a solution is a priori in $W_{1}^{2}$ locally, what is the ensured smoothness of this local solution? It is classical that $f$ must belong to $W_{1}^{2+\varepsilon(k)}$ locally, where $\varepsilon(k)>0$. Finding the best $\varepsilon(k)$ was the key point of the problem by F. Gehring solved by K. Astala [2]. The best $\varepsilon(k)$ turned out to be equal to $\frac{1-k}{k}$. It is not attainable in general.

2) Suppose $\|\mu\|_{\infty}=k<1$. If a solution is a priori in $W_{1}^{q}$ locally (now $q<2$ ), what is the smallest $q$ that ensures that $f \in W_{1}^{2}$ locally (and then, by [2], ensures that $f \in W_{1}^{1+1 / k-\tau}$ for any positive $\tau$ )? The smallest $q$ turns out to be $1+k$. It is attainable (see [30]).

These two questions are intimately related to estimate (0.1). We explain the reason for that. Consider (1.1) in a neighborhood $W$ of the origin, and put $V=\frac{1}{2} W$. Let $\varphi$ be a $C^{\infty}$-function supported on $W$ and equal to 1 on $V$. We set $g:=\varphi f_{\bar{z}}$ and consider $f-\frac{1}{\pi} \int_{\mathbb{C}} \frac{g(\zeta)}{z-\zeta} d A(\zeta)$. Here $d A$ stands for planar Lebesgue measure. Application of the distributional operator $\bar{\partial}$ to the latter expression yields zero in $V$. So, we have a function $h$ analytic in $V$ and such that $f=h+\frac{1}{\pi} \int_{\mathbb{C}} \frac{g(\zeta)}{z-\zeta} d A(\zeta)$. Consequently,

$$
f_{z}=h^{\prime}+T g
$$

in $V$. If we multiply (1.1) by $\varphi$ and use the notation $g:=\varphi f_{\bar{z}}$ and the previous expression for $f_{z}$, we get

$$
g-\mu \varphi T g=r:=\mu \varphi h^{\prime}
$$

in $V$. On $U:=\frac{1}{2} V$ the function $r$ is bounded, and therefore it belongs to any $L^{p}(U, d A)$. Let $M$ denote the operator of multiplication by $\mu \varphi$ in $L^{p}(U, d A),\|M\| \leq k$. We denote $t(p):=\|T\|_{L^{p}(U, d A) \rightarrow L^{p}(U, d A)}$ and consider the identity

$$
(I-M T) g=r
$$

to conclude that the inequality

$$
k t(p)<1
$$

implies that $g \in L^{p}$ in $U$, which is the same as to say that $f \in W_{1}^{p}$ locally. Now we see that (0.1) would imply that for every $p$ with $p<1+\frac{1}{k}$ the solution in question of (1.1) is in $W_{1}^{p}(d A)$ locally.

The above considerations yield also a lower estimate for the norm of $T$ on $L^{p}$. This argument is borrowed from [19]. Suppose that $t(p)$ is strictly smaller than $p-1$. Using (1.2) in the same way as above, we see that if $\|\mu\|_{\infty}=k<1$, then any solution 
of (1.1) that is a priori in $W_{1}^{2}$ locally is in fact in $W_{1}^{1+1 / k+\varepsilon}$ for some $\varepsilon>0$. But it is easy to compute that the function $f(z):=z|z|^{-\frac{2 k}{1+k}}$ satisfies $(1.1)$ with $\mu(z)=-k \frac{z}{\bar{z}}$. Thus, the $L^{\infty}$-norm of $\mu$ is $k$. However, $f$ is not in $W_{1}^{1+1 / k+\varepsilon}$ near the origin. It is not even in $W_{1}^{1+1 / k}$. In fact, it is readily computable that $\left|f_{z}\right|=C(k)|z|^{-\frac{2 k}{1+k}}$, which does not belong to any $L^{p}(d A)$ for $p \geq 1+1 / k$. Thus, $\|T\|_{p} \geq p-1$.

We think that we have presented enough motivation for our interest in the estimation of the $L^{p}$-norm of such a particular Fourier multiplier as $T$, and of related multipliers to be considered here.

\section{§2. LitTlewood-PALEy IDENTITy FOR HEAT EXTENSIONS}

For the Ahlfors-Beurling operator $T$ we can write the identity $T=R_{1}^{2}-R_{2}^{2}+2 i R_{1} R_{2}$, where the $R_{i}$ are the planar Riesz transforms. We fix, say, $R_{1}^{2}$ and two complex-valued test functions $\varphi, \psi \in C_{0}^{\infty}$. We use heat extensions. For a function $f$ on the plane, its heat extension is given by the formula

$$
f(y, t):=\frac{1}{4 \pi t} \iint_{\mathbb{R}^{2}} f(x) \exp \left(-\frac{|x-y|^{2}}{4 t}\right) d x_{1} d x_{2}, \quad(y, t) \in \mathbb{R}_{+}^{3} .
$$

Usually, we employ the same letter to denote a function and its heat extension.

Lemma 2.1. Let $\varphi, \psi \in C_{0}^{\infty}$. Then the integral $\iiint \frac{\partial \varphi}{\partial x_{1}} \cdot \frac{\partial \psi}{\partial x_{1}} d x_{1} d x_{2} d t$ converges absolutely and

$$
\iint R_{1}^{2} \varphi \cdot \psi d x_{1} d x_{2}=-2 \iiint \frac{\partial \varphi}{\partial x_{1}} \cdot \frac{\partial \psi}{\partial x_{1}} d x_{1} d x_{2} d t .
$$

Proof. Actually, the proof of this lemma is trivial. It is based on the fact that a function is an integral of its derivative, and also involves Parseval's formula. Consider complexvalued functions $\varphi, \psi \in C_{0}^{\infty}$ and write

$$
\begin{aligned}
\iint & \psi R_{1}^{2} \varphi d x_{1} d x_{2}=\iint \frac{\xi_{1}^{2}}{\xi_{1}^{2}+\xi_{2}^{2}} \hat{\varphi}\left(\xi_{1}, \xi_{2}\right) \hat{\psi}\left(-\xi_{1},-\xi_{2}\right) d \xi_{1} d \xi_{2} \\
& =2 \iiint_{0}^{\infty} e^{-2 t\left(\xi_{1}^{2}+\xi_{2}^{2}\right)} \xi_{1}^{2} \hat{\varphi}\left(\xi_{1}, \xi_{2}\right) \hat{\psi}\left(-\xi_{1},-\xi_{2}\right) d t d \xi_{1} d \xi_{2} \\
& =-2 \int_{0}^{\infty} \iint i \xi_{1} \hat{\varphi}\left(\xi_{1}, \xi_{2}\right) e^{-t\left(\xi_{1}^{2}+\xi_{2}^{2}\right)} \cdot i \xi_{1} \hat{\psi}\left(-\xi_{1},-\xi_{2}\right) e^{-t\left(\xi_{1}^{2}+\xi_{2}^{2}\right)} d \xi_{1} d \xi_{2} d t \\
& =2 \int_{0}^{\infty} \iint \frac{\partial \varphi}{\partial x_{1}}\left(x_{1}, x_{2}, t\right) \frac{\partial \psi}{\partial x_{1}}\left(x_{1}, x_{2}, t\right) d x_{1} d x_{2} d t \\
& =2 \iiint_{\mathbb{R}_{+}^{3}} \frac{\partial \varphi}{\partial x_{1}}\left(x_{1}, x_{2}, t\right) \frac{\partial \psi}{\partial x_{1}}\left(x_{1}, x_{2}, t\right) d x_{1} d x_{2} d t
\end{aligned}
$$

We have used Parseval's formula twice, and also the absolute convergence of the integrals

$$
\begin{aligned}
& \iiint_{\mathbb{R}_{+}^{3}} e^{-2 t\left(\xi_{1}^{2}+\xi_{2}^{2}\right)} \xi_{1}^{2} \hat{\varphi}\left(\xi_{1}, \xi_{2}\right) \hat{\psi}\left(\xi_{1}, \xi_{2}\right) d \xi_{1} d \xi_{2} d t \\
& \iiint_{\mathbb{R}_{+}^{3}} \frac{\partial \varphi}{\partial x_{1}}\left(x_{1}, x_{2}, t\right) \frac{\partial \psi}{\partial x_{1}}\left(x_{1}, x_{2}, t\right) d x_{1} d x_{2} d t
\end{aligned}
$$

This is obvious for the first integral and easy for the second. We leave this as an exercise for the reader. 


\section{§3. The Bellman function proof of (0.2)}

We warn the reader that sometimes it will be convenient to think that $\mathbb{C}$ is $\mathbb{R}^{2}$, and that the absolute value $|\cdot|$ is the norm $\|\cdot\|$ of a vector in $\mathbb{R}^{2}$.

Let $\varphi, \psi$ be complex-valued test functions in $C_{0}^{\infty}\left(\mathbb{R}^{2}\right)$. We denote their heat extensions to $\mathbb{R}_{+}^{3}$ by the same letters and use Lemma 2.1 . It is easily seen that estimating combinations of $\left\langle R_{i}^{2} \varphi, \psi\right\rangle$ is reduced to estimating integrals that occur in the next theorem. Notice also that if $U_{\rho}$ denotes the operator $U_{\rho} \varphi(z):=f\left(e^{i \rho} z\right)$, then $2 R_{1} R_{2}=U_{\pi / 4}^{*}\left(R_{1}^{2}-R_{2}^{2}\right) U_{\pi / 4}$. Therefore, the proof of (0.2) follows immediately from Theorem 3.1 and Lemma 2.1.

Theorem 3.1. For any complex-valued $\varphi, \psi \in C_{0}^{\infty}$ we have

$$
2 \iiint_{\mathbb{R}_{+}^{3}}\left|\frac{\partial \varphi}{\partial x_{1}}\right|\left|\frac{\partial \psi}{\partial x_{1}}\right| d x_{1} d x_{2} d t+2 \iiint_{\mathbb{R}_{+}^{3}}\left|\frac{\partial \varphi}{\partial x_{2}}\right|\left|\frac{\partial \psi}{\partial x_{2}}\right| d x_{1} d x_{2} d t \leq(p-1)\|\varphi\|_{p}\|\psi\|_{q} .
$$

In particular,

$$
\left\|R_{1}^{2}-R_{2}^{2}\right\|_{p} \leq p-1,\left\|2 R_{1} R_{2}\right\|_{p} \leq p-1 \quad \text { for all } p, 2 \leq p<\infty .
$$

In the proof of Theorem 3.1 we use the following key result. (In what follows, $d^{2} f$ denotes the Hessian form that is the second differential form of $f$.)

Theorem 3.2. For any $p \geq 2$ we define the domain $D_{p}:=\{0<(X, Y, \xi, \eta) \subset \mathbb{R} \times \mathbb{R} \times$ $\left.\mathbb{R}^{2} \times \mathbb{R}^{2}:\|\xi\|^{p}<X,\|\eta\|^{q}<Y\right\}$. Let $K$ be any compact subset of $D_{p}$, and let $\varepsilon$ be an arbitrary positive number. Then there exists a function $B=B_{\varepsilon, p, K}(X, Y, x, y)$ infinitely differentiable in a small neighborhood of $K$ and such that

$$
\begin{aligned}
& \text { 1) } 0 \leq B \leq(1+\varepsilon)(p-1) X^{1 / p} Y^{1 / q}, \\
& \text { 2) }-d^{2} B \geq 2\|d \xi\|\|d \eta\| .
\end{aligned}
$$

We prove Theorem 3.2 later. Now we use it to obtain the proof of Theorem 3.1.

Proof. We consider two functions $\varphi, \psi \in C_{0}^{\infty}$ and take $B=B_{\varepsilon, p, K}$, where a compact set $K$ remains to be chosen.

We are interested in

$$
b(x, t):=B\left(|\varphi|^{p}(x, t),|\psi|^{q}(x, t), \varphi(x, t), \psi(x, t)\right) .
$$

This is a well-defined function, because the Cauchy inequality ensures that the 6-vector

$$
v:=\left(|\varphi|^{p}(x, t),|\psi|^{q}(x, t), \varphi(x, t), \psi(x, t)\right)
$$

lies in $D_{p}$ for any $(x, t) \in \mathbb{R}_{+}^{3}$. Also we can fix any compact subset $M$ of the open set $\mathbb{R}_{+}^{3}$ and guarantee that for $(x, t) \in M$, the vector $v$ lies in some compact set $K$. Indeed, observe that for compactly supported $\varphi, \psi$ the mapping $(x, t) \rightarrow v(x, t)$ takes compact sets in $\mathbb{R}_{+}^{3}$ to compact sets in $D_{p}$. Now we simply take $K$ sufficiently large in accordance with $M$; in our future considerations $M$ will run over larger and larger compact sets in $\mathbb{R}_{+}^{3}$.

We want to apply Green's formula to $b(x, t)$. To do this, we introduce the Green function $G(x, t)$ as in [13]. Taking a sufficiently large cylinder $\Omega:=\Omega_{l}:=D(0, l) \times(0, l)$, we put $\partial^{\prime} \Omega=\partial D(0, l) \times(0, l)$ and consider the following Green function:

$$
\left\{\begin{aligned}
\left(\frac{\partial}{\partial t}+\Delta\right) G_{\Omega} & =-\delta_{0,1} & & \text { in } \Omega, \\
G_{\Omega} & =0 & & \text { on } \partial^{\prime} \Omega, \\
G_{\Omega} & =0 & & \text { if } t=l .
\end{aligned}\right.
$$

Here $\delta_{0,1}$ is the $\delta$-function at the point $(0,1)$. 
Let $k(x, t):=\frac{1}{4 \pi t} e^{-\frac{|x|^{2}}{4 t}}, x:=\left(x_{1}, x_{2}\right)$, be the heat kernel in $\mathbb{R}_{+}^{3}$. The quantity $k(0, t)$ can be understood as the temperature of the point $(0,0)$ on the plane at the time moment $t>0$ if initially (at $t=0$ ) the distribution of temperature coincided with the delta distribution concentrated at $(0,0)$. It is important to keep in mind that

$$
G_{\Omega}(0,0) \rightarrow k(0,1) \quad \text { as } l \rightarrow \infty .
$$

Indeed, it suffices to compare the interpretation of $k(0,1)$ with the fact that $G_{\Omega}(0,0)$ is the temperature at the moment 1 provided the same initial distribution is given but the temperature on $\partial^{\prime} \Omega_{l}$ is kept to be 0 . However, if $l$ is large, it is clear that these two quantities are very close.

We also need the Green function in the cylinder $\Omega\left(R, R^{2}\right)=D(0, l R) \times\left(0, l R^{2}\right)$ :

$$
\left\{\begin{aligned}
\left(\frac{\partial}{\partial t}+\Delta\right) G_{\Omega}^{R} & =-\delta_{0, R^{2}} & & \text { in } \Omega\left(R, R^{2}\right)=D(0, l R) \times\left(0, l R^{2}\right), \\
G_{\Omega}^{R} & =0 & & \text { on } \partial^{\prime} \Omega\left(R, R^{2}\right)=\partial D(0, l R) \times\left(0, l R^{2}\right), \\
G_{\Omega}^{R} & =0 & & \text { if } t=l R^{2} .
\end{aligned}\right.
$$

The following fact is easy.

Lemma 3.3. $G_{\Omega}^{R}(x, t)=\frac{1}{R^{2}} G_{\Omega}\left(x / R, t / R^{2}\right)$.

We are ready to apply Green's formula to $b(x, t)$. First we estimate $b\left(0, R^{2}\right)=$ $B\left(|\varphi|^{p}\left(0, R^{2}\right), \ldots, \psi\left(0, R^{2}\right)\right)$. Using property 1$)$ of $B$ (see Theorem 3.2$)$, we get $(x=$ $\left(x_{1}, x_{2}\right)$ as always, and $\left.1 / p+1 / q=1\right)$ :

$$
b\left(0, R^{2}\right) \leq(1+\varepsilon)(p-1)\left(|\varphi|^{p}\left(0, R^{2}\right)\right)^{1 / p}\left(|\psi|^{q}\left(0, R^{2}\right)\right)^{1 / q} .
$$

Thus,

$$
b\left(0, R^{2}\right) \leq(1+\varepsilon)(p-1)\left(\frac{1}{4 \pi R^{2}} \iint|\varphi|^{p}(x) e^{-\frac{|x|^{2}}{4 R^{2}}}\right)^{1 / p}\left(\frac{1}{4 \pi R^{2}} \iint|\psi|^{q}(x) e^{-\frac{|x|^{2}}{4 R^{2}}}\right)^{1 / q} .
$$

Now, by Green's formula in $C\left(R, R^{2}\right)$, we have

$$
\begin{aligned}
b\left(0, R^{2}\right)= & -\iiint_{\Omega\left(R, R^{2}\right) \cap\{t>\delta\}} b(x, t)\left(\frac{\partial}{\partial t}+\Delta\right) G_{\Omega}^{R}(x, t) d x_{1} d x_{2} d t \\
= & \iiint_{\Omega\left(R, R^{2}\right) \cap\{t>\delta\}} G_{\Omega}^{R}(x, t)\left(\frac{\partial}{\partial t}-\Delta\right) b(x, t) d x_{1} d x_{2} d t \\
& +\iint_{D(0, R)} b(x, \delta) G_{\Omega}^{R}(x, \delta) d x_{1} d x_{2} \\
& +\iint_{\partial^{\prime} \Omega\left(R, R^{2}\right) \cap\{t>\delta\}}\left(\frac{\partial b}{\partial n_{\text {outer }}} G_{\Omega}^{R}-\frac{\partial G_{\Omega}^{R}}{\partial n_{\text {outer }}} b\right) d s d t \\
= & \iiint_{\Omega\left(R, R^{2}\right) \cap\{t>\delta\}} G_{\Omega}^{R}(x, t)\left(\frac{\partial}{\partial t}-\Delta\right) b(x, t) d x_{1} d x_{2} d t \\
& +\iint_{D(0, l R)} b(x, \delta) G_{\Omega}^{R}(x, \delta) d x_{1} d x_{2}+\iint_{\partial^{\prime} \Omega\left(R, R^{2}\right) \cap\{t>\delta\}} \frac{\partial G_{\Omega}^{R}}{\partial n_{\text {inner }}} b d s d t \\
\geq & \iiint_{\Omega\left(R, R^{2}\right) \cap\{t>\delta\}} G_{\Omega}^{R}(x, t)\left(\frac{\partial}{\partial t}-\Delta\right) b(x, t) d x_{1} d x_{2} d t .
\end{aligned}
$$

The last inequality is clear: the double integrals are both nonnegative, because $b$ is nonnegative and because $G_{\Omega}^{R}$ is nonnegative and vanishes on the side boundary. We 
combine the estimates of $b\left(0, R^{2}\right)$ into the following (here $\Omega_{R, \delta}:=\Omega\left(R, R^{2}\right) \cap\{t>\delta\}$ ):

$$
\begin{aligned}
& \iiint_{\Omega_{R, \varepsilon}} G_{\Omega}^{R}(x, t)\left(\frac{\partial}{\partial t}-\Delta\right) b(x, t) \\
& \quad \leq \frac{(1+\varepsilon)(p-1)}{4 \pi R^{2}}\left(\iint|\varphi|^{p}(x) e^{-\frac{|x|^{2}}{4 R^{2}}}\right)^{1 / p}\left(\iint|\psi|^{q}(x) e^{-\frac{|x|^{2}}{4 R^{2}}}\right)^{1 / q} .
\end{aligned}
$$

Fixing $R$ and $\delta>0$, we take the compact set $M=\{(x, t): x \in \operatorname{const}(D(0, l R)), \delta \leq$ $\left.t \leq l R^{2}\right\}$. The vector-valued function $v$ maps $M$ to a compact subset of the domain $D_{p}$. We denote this compact subset by $K$ and choose $B=B_{\varepsilon, p, K}$ as in Theorem 3.2.

The next calculation is simple but it is key to the proof. In it

$$
v=\left(|\varphi|^{p}(x, t),|\psi|^{q}(x, t), \varphi(x, t), \psi(x, t)\right) .
$$

Lemma 3.4. If $(x, t) \in M$, then

$$
\left(\frac{\partial}{\partial t}-\Delta\right) b(x, t)=\left(\left(-d^{2} B\right) \frac{\partial v}{\partial x_{1}}, \frac{\partial v}{\partial x_{1}}\right)_{\mathbb{R}^{6}}+\left(\left(-d^{2} B\right) \frac{\partial v}{\partial x_{2}}, \frac{\partial v}{\partial x_{2}}\right)_{\mathbb{R}^{6}} .
$$

Proof.

$$
\begin{aligned}
\frac{\partial}{\partial t} b & =\left(\nabla B, \frac{\partial v}{\partial t}\right)_{\mathbb{R}^{6}}, \\
\Delta b & =\left(\left(d^{2} B\right) \frac{\partial v}{\partial x_{1}}, \frac{\partial v}{\partial x_{1}}\right)_{\mathbb{R}^{6}}+\left(\left(d^{2} B\right) \frac{\partial v}{\partial x_{2}}, \frac{\partial v}{\partial x_{2}}\right)_{\mathbb{R}^{6}}+(\nabla B, \Delta v)_{\mathbb{R}^{6}} .
\end{aligned}
$$

(Merely, we have used the chain rule.) Now,

$$
\left(\frac{\partial}{\partial t}-\Delta\right) b=\left(\nabla B, \frac{\partial v}{\partial t}-\Delta v\right)_{\mathbb{R}^{6}}-\left(\left(d^{2} B\right) \frac{\partial v}{\partial x_{1}}, \frac{\partial v}{\partial x_{1}}\right)_{\mathbb{R}^{6}}-\left(\left(d^{2} B\right) \frac{\partial v}{\partial x_{2}}, \frac{\partial v}{\partial x_{2}}\right)_{\mathbb{R}^{6}} .
$$

However, the first term is zero because all entries of the vector $v$ are solutions of the heat equation.

By Theorem 3.2, in $M$ we have

$$
-d^{2} B(X, Y, \xi, \eta) \geq 2\|d \xi\|\|d \eta\| .
$$

For $(x, t) \in M$, Lemma 3.4 yields

$$
\left(\frac{\partial}{\partial t}-\Delta\right) b(x, t) \geq 2\left|\frac{\partial \varphi}{\partial x_{1}}\right|\left|\frac{\partial \psi}{\partial x_{1}}\right|+\left|\frac{\partial \varphi}{\partial x_{2}}\right|\left|\frac{\partial \psi}{\partial x_{2}}\right| .
$$

Combining (3.2) and (3.4), we get

$$
\begin{aligned}
& 2 \iiint_{M} G_{\Omega}^{R}(x, t)\left(\left|\frac{\partial \varphi}{\partial x_{1}}\right|\left|\frac{\partial \psi}{\partial x_{1}}\right|+\left|\frac{\partial \varphi}{\partial x_{2}}\right|\left|\frac{\partial \psi}{\partial x_{2}}\right|\right) \\
& \quad \leq \frac{(1+\varepsilon)(p-1)}{4 \pi R^{2}}\left(\iint|\varphi|^{p}(x)\right)^{1 / p}\left(\iint|\psi|^{q}(x)\right)^{1 / q} .
\end{aligned}
$$

Now it is time to use Lemma 3.3. So, (3.5) implies the inequality

$$
\begin{aligned}
& 2 \iiint_{M} G_{\Omega}\left(\frac{x}{R}, \frac{t}{R^{2}}\right)\left(\left|\frac{\partial \varphi}{\partial x_{1}}\right|\left|\frac{\partial \psi}{\partial x_{1}}\right|+\left|\frac{\partial \varphi}{\partial x_{2}}\right|\left|\frac{\partial \psi}{\partial x_{2}}\right|\right) \\
& \leq \frac{(1+\varepsilon)(p-1)}{4 \pi}\left(\iint|\varphi|^{p}(x)\right)^{1 / p}\left(\iint|\psi|^{q}(x)\right)^{1 / q} .
\end{aligned}
$$


But $M=\left\{(x, t): x \in \operatorname{const}(D(0, R)), \delta \leq t \leq R^{2}\right\}$. We fix any compact $M_{0}$ in $\mathbb{R}_{+}^{3}$ and choose $R$ and $\delta>0$ in such a way that $M_{0} \subset M$. Next, we restrict the integration in (3.6) to $M_{0}$ and let $R \rightarrow \infty$. Since

$$
G_{\Omega}\left(x / R, t / R^{2}\right) \rightarrow G_{\Omega}(0,0)
$$

we obtain

$$
\begin{aligned}
& 2 G_{\Omega_{l}}(0,0) \iiint_{M_{0}}\left(\left|\frac{\partial \varphi}{\partial x_{1}}\right|\left|\frac{\partial \psi}{\partial x_{1}}\right|+\left|\frac{\partial \varphi}{\partial x_{2}}\right|\left|\frac{\partial \psi}{\partial x_{2}}\right|\right) \\
& \quad \leq \frac{(1+\varepsilon)(p-1)}{4 \pi}\left(\iint|\varphi|^{p}(x)\right)^{1 / p}\left(\iint|\psi|^{q}(x)\right)^{1 / q} .
\end{aligned}
$$

Now it is time to let $\Omega=\Omega_{l}$ tend to $\mathbb{R}_{+}^{3}$ by making $l$ go to infinity. By (3.1), we conclude that $G_{\Omega_{l}}(0,0) \rightarrow \frac{1}{4 \pi}$. Then (3.7) becomes

$$
\begin{aligned}
& 2 \iiint_{M_{0}}\left(\left|\frac{\partial \varphi}{\partial x_{1}}\right|\left|\frac{\partial \psi}{\partial x_{1}}\right|+\left|\frac{\partial \varphi}{\partial x_{2}}\right|\left|\frac{\partial \psi}{\partial x_{2}}\right|\right) \\
& \quad \leq(1+\varepsilon)(p-1)\left(\iint|\varphi|^{p}(x)\right)^{1 / p}\left(\iint|\psi|^{q}(x)\right)^{1 / q} .
\end{aligned}
$$

But $M_{0}$ is an arbitrary compact set in the upper half-space, and $\varepsilon$ is an arbitrary positive number. Therefore,

$$
\begin{aligned}
& 2 \iiint_{\mathbb{R}_{+}^{3}}\left(\left|\frac{\partial \varphi}{\partial x_{1}}\right|\left|\frac{\partial \psi}{\partial x_{1}}\right|+\left|\frac{\partial \varphi}{\partial x_{2}}\right|\left|\frac{\partial \psi}{\partial x_{2}}\right|\right) \\
& \quad \leq(p-1)\left(\iint|\varphi|^{p}(x)\right)^{1 / p}\left(\iint|\psi|^{q}(x)\right)^{1 / q} .
\end{aligned}
$$

This proves Theorem 3.1 .

\section{§4. The existence of a Bellman function. Proof of Theorem 3.2}

We start with a simple "model" operator $T_{\sigma}$. To define it, we let $\mathcal{D}$ denote a family of dyadic intervals on the line. To each $I \in \mathcal{D}$ we assign its Haar function: $h_{I}=1 / \sqrt{|I|}$ on $I_{+}$and $h_{I}=-1 / \sqrt{|I|}$ on $I_{-}$, where $I_{+}$and $I_{-}$are the right half and the left half of $I$, respectively. Every nice complex-valued function (continuous with compact support on one of $I$ 's, say on $[0,1])$ can be written as its Haar series: $f=\sum_{I}\left(f, h_{I}\right) h_{I}$. Consider the operator $T_{\sigma} f=\sum_{I} \sigma_{I}\left(f, h_{I}\right) h_{I}$, where $\sigma_{I}$ is an arbitrary sequence of complex numbers with absolute value 1 . Notation: we use $\langle f\rangle_{I}$ to denote $\frac{1}{|I|} \int_{I} f d x$.

The logic will be as follows. We want to get a sharp estimate of $\left\|T_{\sigma}\right\|_{L^{p} \rightarrow L^{p}}$ in terms of $p$. This problem has been solved by Burkholder. In [10] he found that $(p \geq 2)$

$$
\sup _{\sigma}\left\|T_{\sigma}\right\|_{p} \leq p-1 \text {. }
$$

He proved (4.1) by constructing a certain function of two real variables (actually, another Bellman function) that has certain convexity and size properties. The reader is referred to the papers by Burkholder [10, 11], or to the book by D. Stroock [32] for the description of his approach. In particular, the following is written about (4.1) in [32] p. 344]: "Quite recently Burkholder has discovered the right argument: ... it is completely elementary. Unfortunately, it is also completely opaque. Indeed, his new argument is nothing but an elementary verification that he has got the right answer; it gives no hint about how he came to that answer". Further on "for those who want to know the secret 
behind his proof, Burkholder has written an explanation in his article" [10]. Here is Burkholder's function $(p \geq 2)$ :

$$
b(x, y)=(|x|-(p-1)|y|)(|x|+|y|)^{p-1} .
$$

Actually, a stochastic Bellman PDE readily explains the way to write this function, and this was done, for example, in [33].

We wish we could use this Bellman function of Burkholder in our problem. But we are unable to do that. The reason is simple. The variables in Burkholder's function stand for certain martingales, which in his case are related to each other: one is subordinate to the other. In our case we replace this variables not by martingales but by functions: the first is $R_{1}^{2} \varphi$, and the second is $\varphi$, where $\varphi$ is a test function. There is no subordination here. The only differential relation between these two functions (actually, between their heat extensions) is the following:

$$
\frac{\partial}{\partial t} R_{1}^{2} \varphi=\frac{\partial^{2}}{\partial x_{1}^{2}} \varphi
$$

This is a second order differential identity and, as such, it is in no relationship with the subordination property, which interplayed so essentially with a very special convexity property of the Burkholder function (see [10]). It would have been related to subordination (and then to convexity), should it be a differential identity of the first order. What we mean can be illustrated by the following oversimplified example. Obviously, the composition of a convex function $a$ with a linear function $l$ is convex, but the composition of a convex $a$ and a convex $l$ may fail to be convex $\left(a(x)=e^{-x}, l(x)=x^{2}\right)$. That is exactly the obstruction to using Burkholder's function and composing it with our second order Riesz transforms.

We do not see the way to win over this difficulty. We prefer another approach, which follows the approach in [30].

The idea: we formulate Burkholder's inequality in an equivalent form (simply in its dual form). The resulting inequality generates another Bellman function. This will be our $B$ in Theorem 3.2 .

As has already been said, we shall use the following lemma due to Burkholder.

Lemma 4.1. Let $H$ be a separable Hilbert space. Suppose $\left(X_{n}, F_{n}, P\right)$ and $\left(Y_{n}, F_{n}, P\right)$ are $H$-valued martingales. If

$$
\left\|X_{0}(\omega)\right\|_{H} \leq\left\|Y_{0}(\omega)\right\|_{H}, \quad\left\|X_{n}(\omega)-X_{n-1}(\omega)\right\|_{H} \leq\left\|Y_{n}(\omega)-Y_{n-1}(\omega)\right\|_{H}
$$

for almost every $\omega$ and all $n$, then

$$
\left\|X_{n}\right\|_{L^{p}(P, H)} \leq \max (p-1,1 /(p-1))\left\|Y_{n}\right\|_{L^{p}(P, H)}
$$

for each $p \in(1, \infty)$.

From the lemma we can easily deduce the following theorem.

Theorem 4.2. Suppose $J \in \mathcal{D}, f \in L^{p}(J)$, and $g \in L^{p^{\prime}}(J)$. Let $p \geq 2$. Then

$$
\frac{1}{4} \frac{1}{|J|} \sum_{I \in \mathcal{D}, I \subset J}\left|\langle f\rangle_{I_{+}}-\langle f\rangle_{I_{-}}\right|\left|\langle g\rangle_{I_{+}}-\langle g\rangle_{I_{-}}\right||I| \leq(p-1)\left\langle|f|^{p}\right\rangle_{J}^{1 / p}\left\langle|g|^{p^{\prime}}\right\rangle_{J}^{1 / p^{\prime}} .
$$

Proof. Without loss of generality, let $J=[0,1]$. Let $F_{n}$ be the $\sigma$-algebra generated by all dyadic subintervals in $J$ of length at least $2^{-n}$. Consider $\omega \in[0,1]$ and put

$$
Y_{n}(\omega):=\sum_{I \subset J,|I| \geq 2^{-n}}\left(f, h_{I}\right) h_{I}(\omega)
$$


We fix any sequence of complex numbers $\sigma_{I}=e^{i \alpha_{I}}, \alpha_{I} \in \mathbb{R}$, and consider

$$
X_{n}(\omega):=\sum_{I \subset J,|I| \geq 2^{-n}} \sigma_{I}\left(f, h_{I}\right) h_{I}(\omega) .
$$

Both $\left(X_{n}, F_{n}, d x\right)$ and $\left(Y_{n}, F_{n}, d x\right)$ are martingales. Clearly,

$$
Y_{n}-Y_{n-1}=\sum_{I \subset J,|I|=2^{-n}}\left(f, h_{I}\right) h_{I},
$$

and

$$
X_{n}-X_{n-1}:=\sum_{I \subset J,|I|=2^{-n}} \sigma_{I}\left(f, h_{I}\right) h_{I} .
$$

These martingales satisfy the assumptions of Burkholder's lemma. The Hilbert space $H=\mathbb{R}^{2}$ is identified naturally with $\mathbb{C}$. Now $\|f\|_{L^{p}}=\lim _{n \rightarrow \infty}\left\|Y_{n}\right\|_{L^{p}(H)},\left\|T_{\sigma} f\right\|_{L^{p}}=$ $\lim _{n \rightarrow \infty}\left\|X_{n}\right\|_{L^{p}(H)}$; Burkholder's lemma implies that

$$
\left\|T_{\sigma} f\right\|_{L^{p}} \leq(p-1)\|f\|_{L^{p}}
$$

for $p \geq 2$ and for any sequence $\sigma$ as above.

We reformulate $(4.2)$ as $\left|\left(T_{\sigma} f, g\right)\right| \leq(p-1)\|f\|_{L^{p}}\|g\|_{L^{p^{\prime}}}$. Now the definition of $T_{\sigma}$ implies

$$
\frac{1}{|J|}\left|\sum_{I} \sigma_{I}\left(f, h_{I}\right) \overline{\left(g, h_{I}\right)}\right| \leq(p-1)\left\langle|f|^{p}\right\rangle_{J}^{1 / p}\left\langle|g|^{p^{\prime}}\right\rangle_{J}^{1 / p^{\prime}}, \quad \sigma_{I}=e^{i \alpha_{I}}
$$

Notice that $\left(f, h_{I}\right)=\frac{1}{2} \sqrt{|I|}\left(\langle f\rangle_{I_{+}}-\langle f\rangle_{I_{-}}\right)$. Since we also may vary $\sigma_{I}$, the theorem follows.

Theorem 4.3. In the domain $G=\left\{(\Phi, \Psi, \phi, \psi) \in \mathbb{R} \times \mathbb{R} \times \mathbb{C} \times \mathbb{C}:|\phi|^{p}<\Phi,|\psi|^{p^{\prime}}<\Psi\right\}$, there exists a function $B(\Phi, \Psi, \phi, \psi)$ such that for any quadruples $a=(\Phi, \Psi, \phi, \psi), a_{-}=$ $\left(\Phi_{-}, \Psi_{-}, \phi_{-}, \psi_{-}\right)$, and $a_{+}=\left(\Phi_{+}, \Psi_{+}, \phi_{+}, \psi_{+}\right)$with $a=\frac{a_{-}+a_{+}}{2}$ we have

$$
B(a)-\frac{1}{2}\left(B\left(a_{-}\right)+B\left(a_{+}\right)\right) \geq \frac{1}{4}\left|\phi_{-}-\phi_{+}\right|\left|\psi_{-}-\psi_{+}\right| .
$$

Also,

everywhere in $G$.

$$
0 \leq B(a) \leq(p-1) \Phi^{1 / p} \Psi^{1 / p^{\prime}}
$$

For every compact subset $K$ in $G$ we can find an infinitely smooth function $B_{K}$ on $K$ such that the first estimate is fulfilled. Consider $\phi=\xi_{1}+i \eta_{1}, \psi=\xi_{2}+i \eta_{2}$, where the $\xi$ 's and $\eta$ 's are real. If we view $B_{K}$ as a function of 6 real variables, then we can consider its $6 \times 6$ Jacobi matrix and the corresponding second differential form, i.e., the Hessian. Then the Hessian of $B_{K}$ must satisfy the following inequality:

$$
-d^{2} B_{K} \geq 2|d \phi||d \psi|=2\left(\left(d \xi_{1}\right)^{2}+\left(d \eta_{1}\right)^{2}\right)^{1 / 2}\left(\left(d \xi_{2}\right)^{2}+\left(d \eta_{2}\right)^{2}\right)^{1 / 2} .
$$

At the same time, for any positive $\varepsilon, B_{K}$ can be chosen to satisfy

$$
0 \leq B_{K}(a) \leq(1+\varepsilon)(p-1) \Phi^{1 / p} \Psi^{1 / p^{\prime}} .
$$

Proof. Fix $(\Phi, \Psi, \phi, \psi) \in G$ and consider all complex-valued functions $f, g$ on $J$ such that $\Phi=\left\langle|f|^{p}\right\rangle_{J}, \Psi=\left\langle|g|^{p^{\prime}}\right\rangle_{J}, \phi=\langle f\rangle_{J}, \psi=\langle g\rangle_{J}$. Let

$$
B(\Phi, \Psi, \phi, \psi):=\sup \left\{\frac{1}{4} \frac{1}{|J|} \sum_{I \in \mathcal{D}, I \subset J}\left|\langle f\rangle_{I_{+}}-\langle f\rangle_{I_{-}}\right|\left|\langle g\rangle_{I_{+}}-\langle g\rangle_{I_{-}}\right||I|\right\},
$$

where the supremum is taken over all $f$ and $g$ as above. This supremum does not depend on the interval $J$. This observation helps to prove the first inequality $B(a)-\frac{1}{2}\left(B\left(a_{-}\right)+\right.$ $\left.B\left(a_{+}\right)\right) \geq \frac{1}{4}\left|\phi_{-}-\phi_{+}\right|\left|\psi_{-}-\psi_{+}\right|$exactly as this is done in any paper on Bellman functions. 
On the other hand, the second inequality $0 \leq B(a) \leq(p-1) \Phi^{1 / p} \Psi^{1 / p^{\prime}}$ has already been proved - this is the claim of Theorem 4.2 .

If we fix a compact set $K$, we can also fix a very small $\varepsilon$ (much smaller than the distance from $K$ to the boundary of $G$ ), and we can consider $\frac{1}{\varepsilon^{6}} S\left(\frac{a}{\varepsilon}\right)$, where $S$ is a $C_{0}^{\infty}$ function supported on the unit ball of $\mathbb{R} \times \mathbb{R} \times \mathbb{C} \times \mathbb{C}=\mathbb{R}^{6}$ centered at zero. It remains to mollify $B$ by convolving it with $\frac{1}{\varepsilon^{6}} S\left(\frac{a}{\varepsilon}\right)$. The concavity inequality will be satisfied with no change for the new function. The size inequality can become $\left(1+C_{K} \varepsilon\right)$ times worse.

\section{REFERENCES}

[1] K. Astala, T. Iwaniec, and E. Saksman, Beltrami operators in the plane, Duke Math. J. 107 (2001), no. 1, 27-56. MR 1815249 (2001m:30021)

[2] K. Astala, Area distortion of quasiconformal mappings, Acta Math. 173 (1994), 37-60. MR 1294669 (95m:30028b)

[3] R. Bañuelos and G. Wang, Sharp inequalities for martingales with applications to the BeurlingAhlfors and Riesz transforms, Duke Math. J. 80 (1995), no. 3, 575-600. MR 1370109 (96k:60108)

[4] R. Bañuelos and P. Méndez-Hernández, Sharp inequalities for Riesz transforms and space time Brownian motion, Indiana Univ. Math. J. (to appear). MR 2001941 (2004h:60067)

[5] B. V. Bojarski, Homeomorphic solutions of Beltrami systems, Dokl. Akad. Nauk SSSR (N.S.) 102 (1955), no. 4, 661-664. (Russian) MR 0071620 (17:157a)

[6] _ Generalized solutions of a system of differential equations of the 1st order and of elliptic type with discontinuous coefficients, Mat. Sb. (N.S.) 43 (1957), no. 4, 451-503. (Russian) MR 0106324 (21:5058)

[7] _ Quasiconformal mappings and general structural properties of systems of nonlinear equations elliptic in the sense of Lavrent'ev, Sympos. Math., vol. 18, Academic Press, London, 1976, pp. 485-499. MR 0507823 (58:22542)

[8] B. V. Bojarski and T. Iwaniec, Quasiconformal mappings and non-linear elliptic equations in two variables. I, II, Bull. Acad. Polon. Sci. Sér. Sci. Math. Astronom. Phys. 22 (1974), 473-484. MR 0364856 (51:1110)

[9] R. Bañuelos and P. Méndez-Hernández, Sharp inequalities for heat kernels of Schrödinger operators and applications to spectral gaps, J. Funct. Anal. 176 (2000), no. 2, 368-399. MR 1784420 (2001f:35096)

[10] D. L. Burkholder, Explorations in martingale theory and its applications, École d'Eté de Probabilités de Saint-Flour XIX-1989, Lecture Notes in Math., vol. 1464, Springer, Berlin, 1991, pp. 1-66. MR 1108183 (92m:60037)

[11] , Boundary value problems and sharp inequalities for martingale transforms, Ann. Probab. 12 (1984), 647-702. MR 0744226 (86b:60080)

[12] S. Buckley, Estimates for operator norms on weighted spaces and reverse Jensen inequalities, Trans. Amer. Math. Soc. 340 (1993), no. 1, 253-272. MR 1124164 (94a:42011)

[13] R. Fefferman, C. Kenig, and J. Pipher, The theory of weights and the Dirichlet problem for elliptic equations, Ann. of Math. (2) 134 (1991), no. 1, 65-124. MR 1114608 (93h:31010)

[14] J. Garcia-Cuerva and J. Rubio de Francia, Weighted norm inequalities and related topics, North-Holland Math. Stud., vol. 116, North-Holland Publishing Co., Amsterdam etc., 1985. MR 0807149 (87d:42023)

[15] F. W. Gehring, Open problems, Proceedings of Roumanian-Finnish Seminar on Teichmüller Spaces and Quasiconformal Mappings, 1969, p. 306.

[16] The $L^{p}$-integrability of the partial derivatives of a quasiconformal mapping, Acta Math. 130 (1973), 265-277. MR 0402038 (53:5861)

[17] _ Topics in quasiconformal mappings, Proceedings of the International Congress of Mathematicians, Vol. 1, 2 (Berkeley, CA, 1986), Amer. Math. Soc., Providence, RI, 1987, pp. 62-80. MR 0934216 (89c:30051)

[18] F. W. Gehring and E. Reich, Area distortion under quasiconformal mappings, Ann. Acad. Sci. Fenn. Ser A I No. 388 (1966), 1-15. MR 0201635 (34:1517)

[19] T. Iwaniec, Extremal inequalities in Sobolev spaces and quasiconformal mappings, Z. Anal. Anwendungen 1 (1982), 1-16. MR 0719167 (85g:30027)

[20] — The best constant in a BMO-inequality for the Beurling-Ahlfors transform, Michigan Math. J. 33 (1986), 387-394. MR 0856530 (88b:42024) 
[21] _ Hilbert transform in the complex plane and area inequalities for certain quadratic differentials, Michigan Math. J. 34 (1987), 407-434. MR 0911814 (89a:42025)

[22] _ $L^{p}$-theory of quasiregular mappings, Quasiconformal Space Mappings, Lecture Notes in Math., vol. 1508, Springer, Berlin, 1992, pp. 39-64. MR 1187088

[23] T. Iwaniec and G. Martin, Quasiregular mappings in even dimensions, Acta Math. 170 (1993), no. 1, 29-81. MR 1208562 (94m:30046)

[24] _ Riesz transforms and related singular integrals, J. Reine Angew. Math. 473 (1996), 25-57. MR 1390681 (97k:42033)

[25] St. Petermichl and J. Wittwer, A sharp weighted estimate on the norm of Hilbert transform via invariant $A_{2}$ characteristic of the weight, Preprint, Michigan State Univ., 2000.

[26] J. Wittwer, Thesis, Univ. Chicago, 2000.

[27] O. Lehto, Quasiconformal mappings and singular integrals, Sympos. Math., vol. 18, Academic Press, London, 1976, pp. 429-453. MR 0492241 (58:11387)

[28] O. Lehto and K. Virtanen, Quasiconformal mappings in the plane, Grundlehren Math. Wiss., vol. 126, Springer-Verlag, New York etc., 1973. MR 0344463 (49:9202)

[29] F. Nazarov, S. Treil, and A. Volberg, The Bellman functions and two-weght inequalities for Haar multipliers, J. Amer. Math. Soc. 12 (1999), 909-928. MR 1685781 (2000k:42009)

[30] S. Petermichl and A. Volberg, Heating of the Ahlfors-Beurling operator: weakly quasiregular maps on the plane are quasiregular, Duke Math. J. 112 (2002), no. 2, 281-305. MR 1894362 (2003d:42025)

[31] E. Stein, Harmonic analysis: real-variable methods, orthogonality, and oscillatory integrals, Princeton Math. Ser., vol. 43, Monogr. Harmon. Anal., vol. III, Princeton Univ. Press, Princeton, NJ, 1993. MR 1232192 (95c:42002)

[32] D. W. Stroock, Probability theory, an analytic view, Cambridge Univ. Press, Cambridge, 1993. MR 1267569 (95f:60003)

[33] A. Volberg, Bellman approach to some problems in harmonic analysis, Séminaire sur les Équations aux Dérivées Partielles, École Polytech., 2002, Exp. No. XX.

Michigan State University, East Lansing, Michigan, USA, and Equipe d'Analyse Université Paris Vi, 4 Place Jussieu, 75252 Paris cédex 05, France

E-mail address: volberg@math.msu.edu

Michigan State University, East Lansing, Michigan, USA

E-mail address: fedja@math.msu.edu

Received 20/DEC/2002

Translated by THE AUTHORS 